Fig 1A PFOS $(\mu \mathrm{M})$

C $10 \quad 50 \quad 100$

Beclin $\frac{1 \rightarrow}{55}$

$6 \mathrm{~h}$

$\mathrm{LC} 3 \mathrm{~B}-\mathrm{I} \underset{ }{\mathrm{LC} 3 \mathrm{~B}-\mathrm{II}} \stackrel{\rightarrow}{\rightarrow}$
$\rightarrow$

10

p62

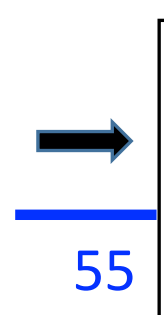

$\beta$-actin $\stackrel{43}{\rightarrow}$ 
Fig 1B_1
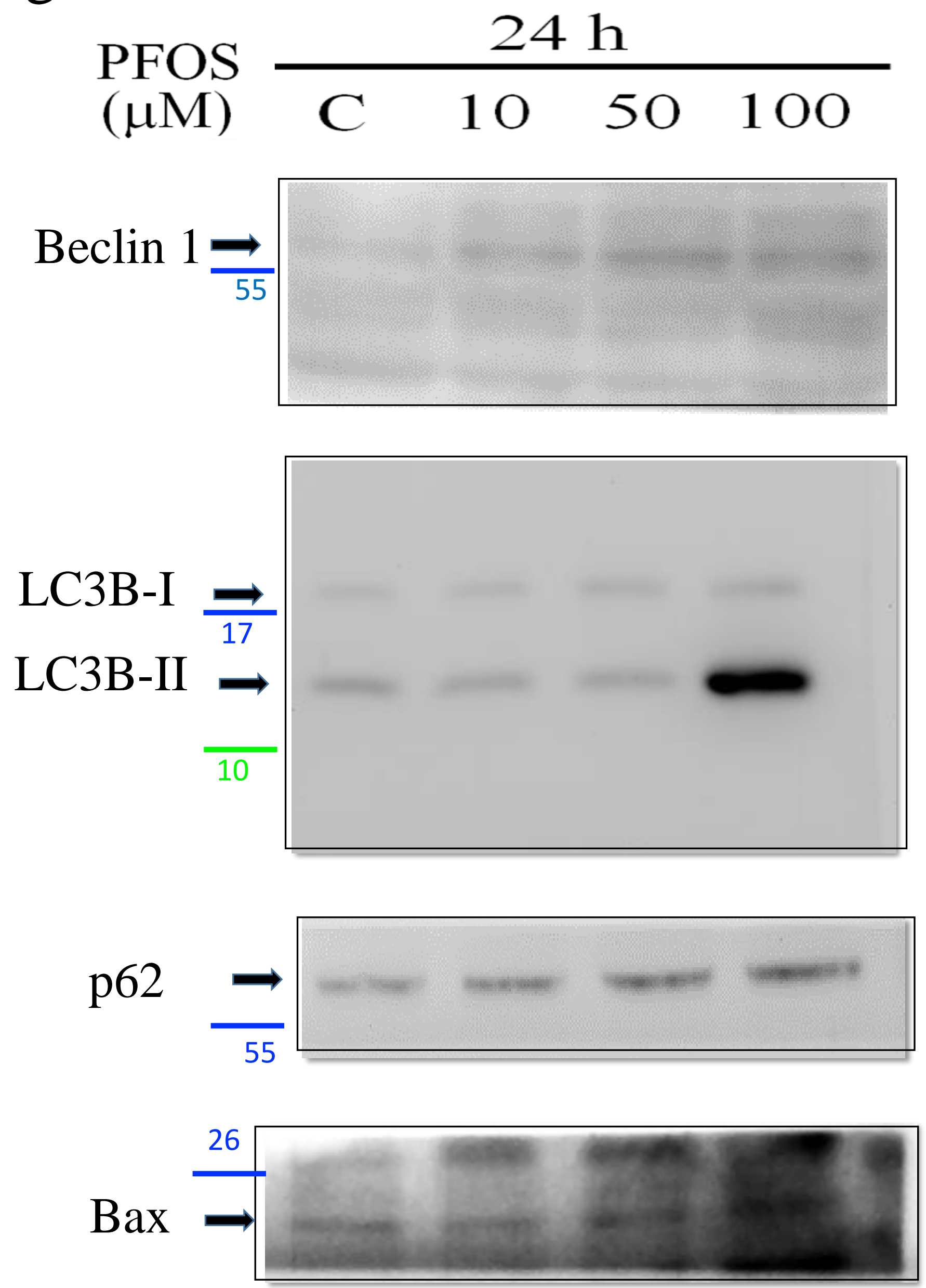
Fig 1B_2

PFOS

$24 \mathrm{~h}$

( $\mu \mathrm{M})$

C

10

50

100

Caspase $3 \stackrel{34}{\rightarrow}$

26

Cleaved

Caspase $3 \overrightarrow{17}$

PARP

Cleaved

$\stackrel{130}{\overrightarrow{95}}$

PARP

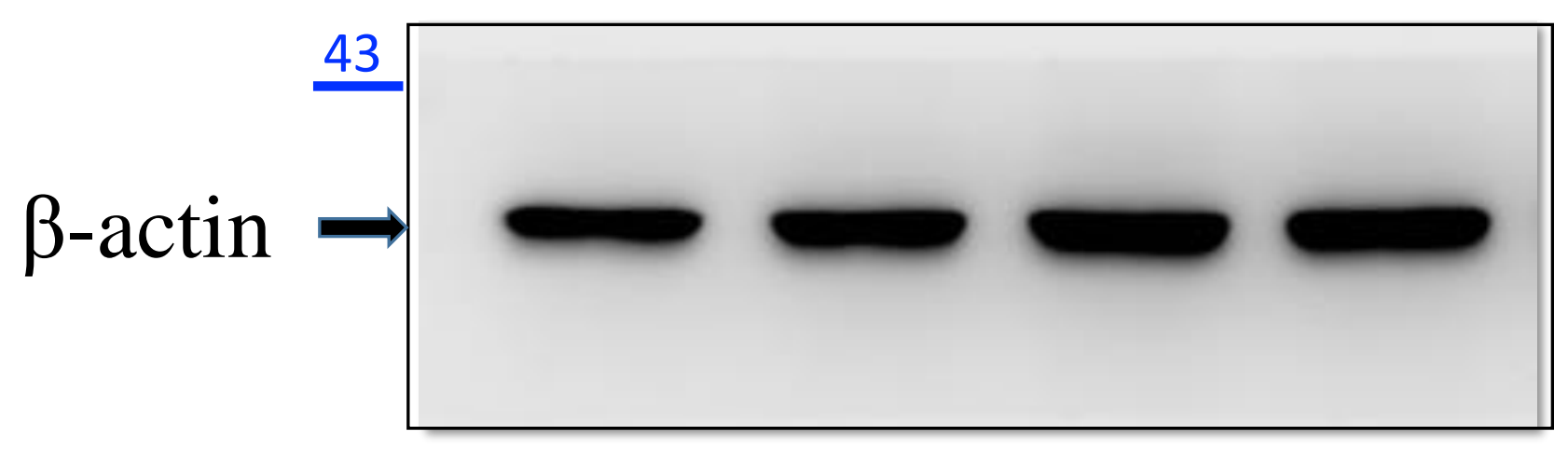




\section{Fig 2A_1}

$24 \mathrm{~h}$

$\begin{array}{lllll}\operatorname{PFOS}(100 \mu \mathrm{M}) & - & + & + & - \\ 3-M A(1 \mathrm{mM}) & - & - & + & +\end{array}$

Beclin 1

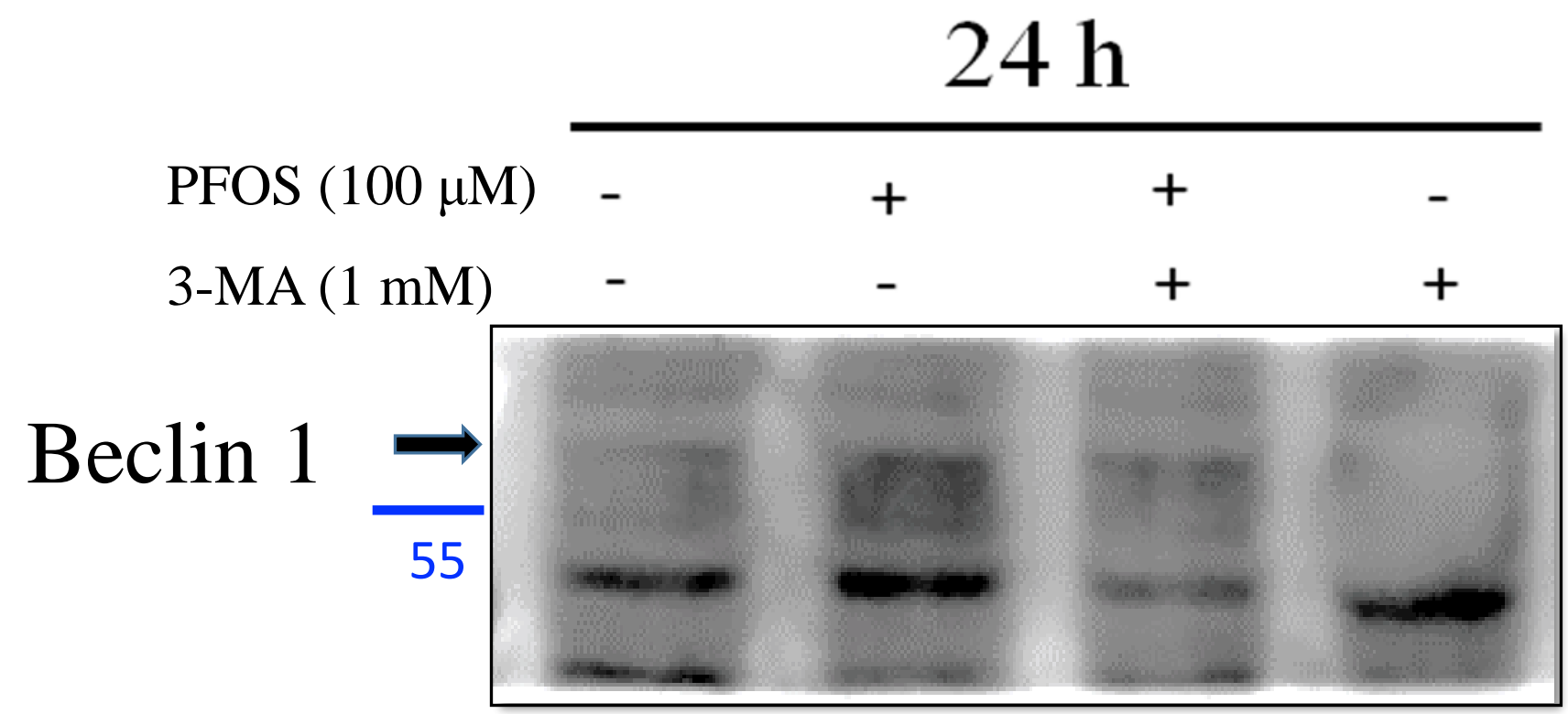

LC3B-I $\overrightarrow{\overrightarrow{17}}$
LC3B-II

10

p62
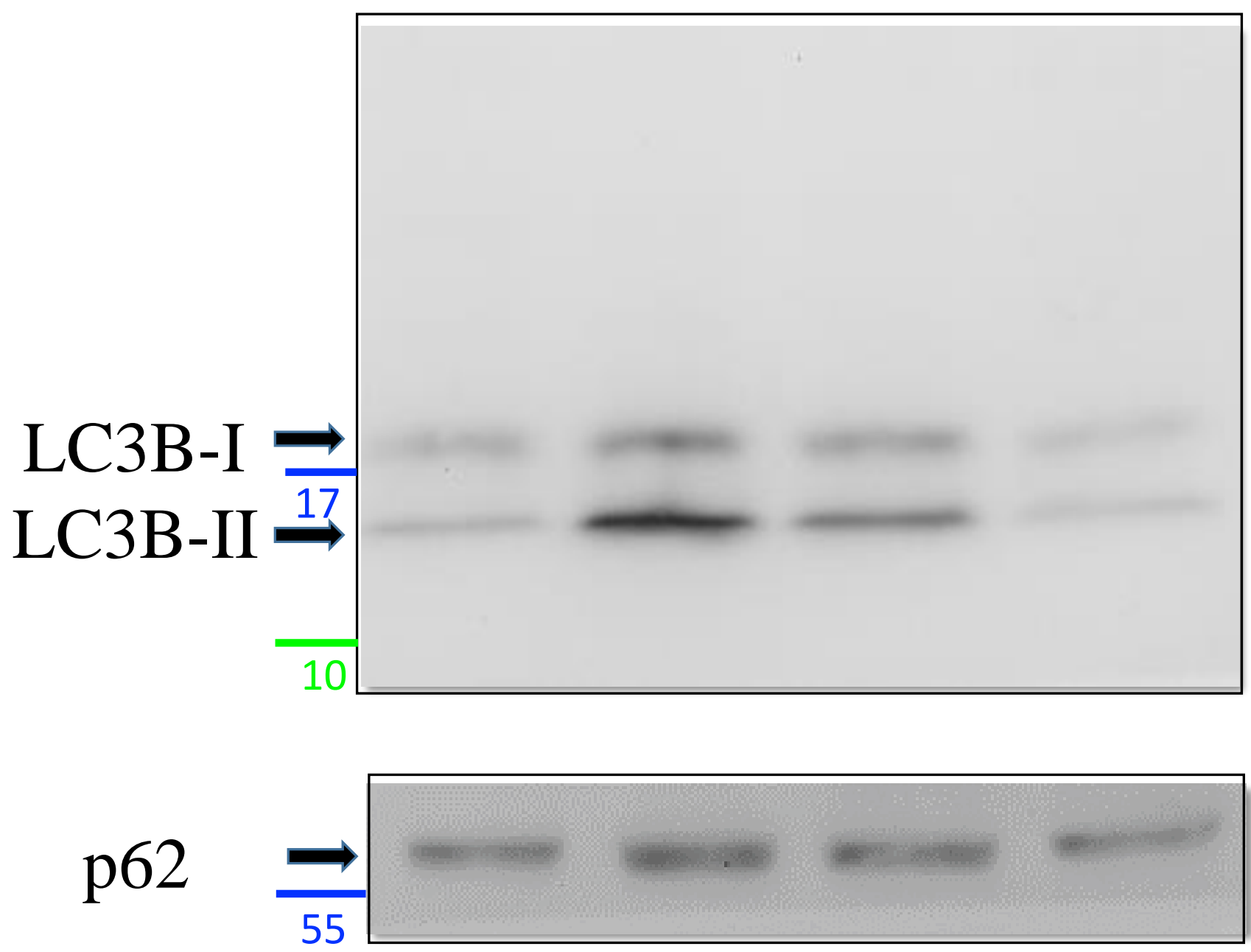
Fig 2A_2

\section{$24 \mathrm{~h}$}

PFOS $(100 \mu \mathrm{M}) \quad-$

3-MA (1 mM)
$+$

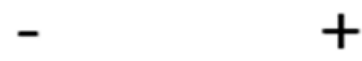

$+$

$+$
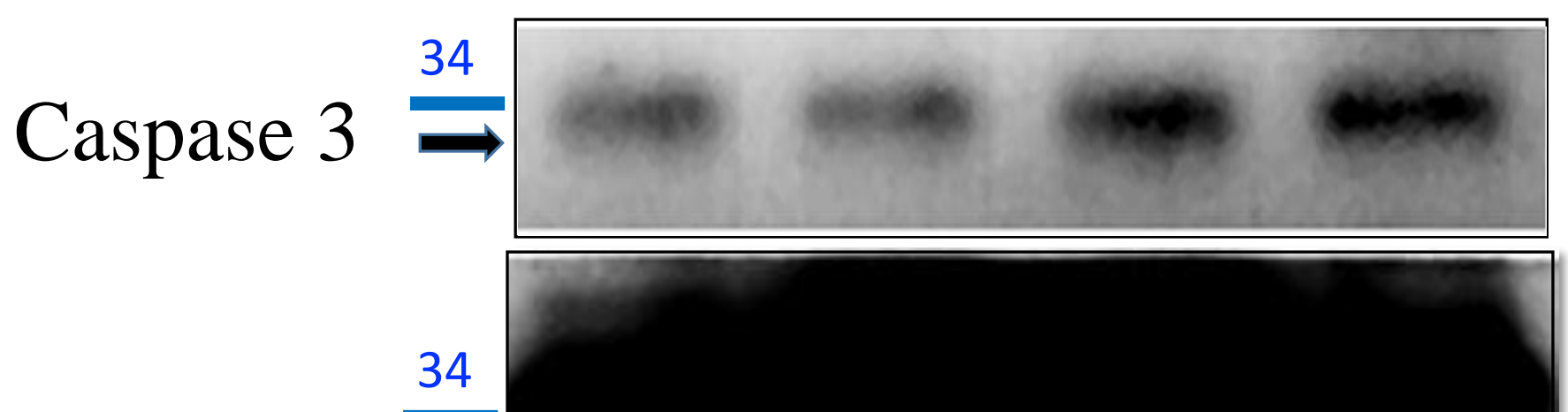

Caspase 3

Cleaved

Caspase 3

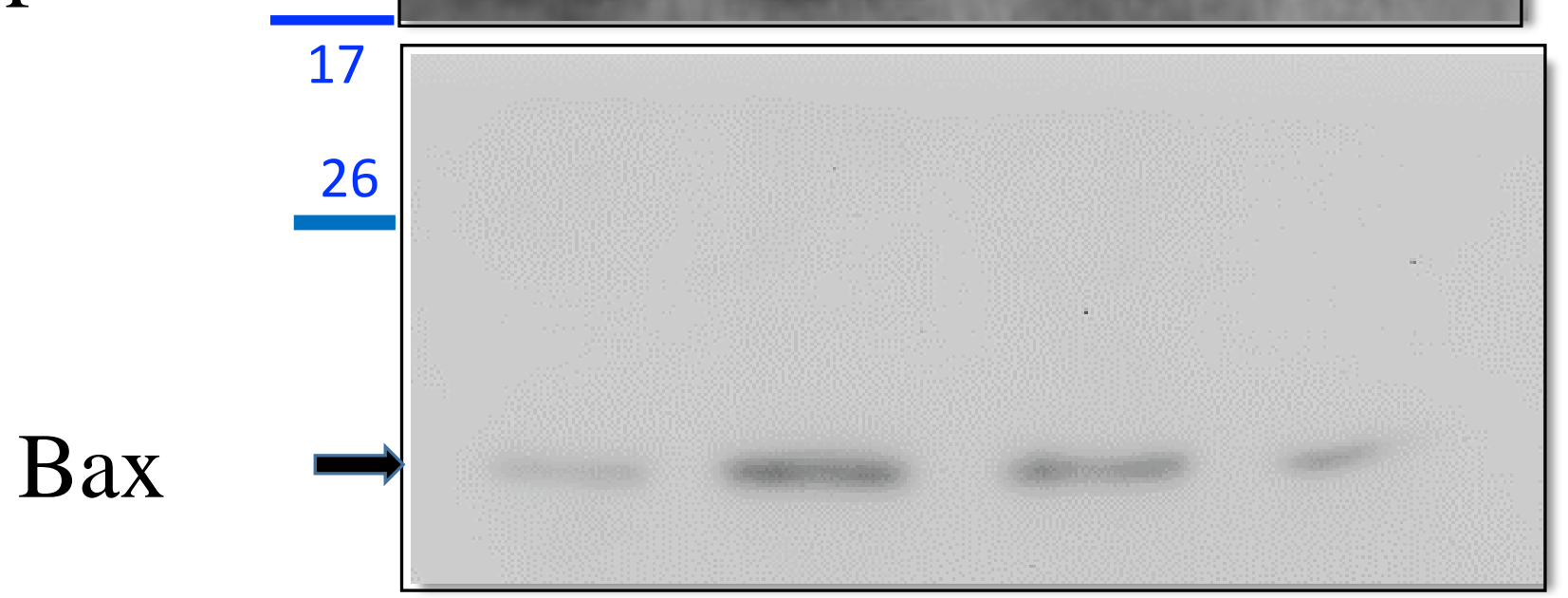

$\beta$-actin $\stackrel{43}{\longrightarrow}$ 
Fig 3B

PFOS $(100 \mu \mathrm{M})$ -

$\begin{array}{lll}+ & + & - \\ - & + & +\end{array}$

LC3B-I $\rightarrow$

LC3B-II $\stackrel{\overrightarrow{17}}{\longrightarrow}$

$\beta$-actin $\stackrel{43}{\longrightarrow}$

$6 \mathrm{~h}$

$24 \mathrm{~h}$

LC3B-I

LC3B-II $\stackrel{\overrightarrow{17}}{\longrightarrow}$ 10

$\beta-\operatorname{actin} \stackrel{43}{\longrightarrow}$ 
Fig 5A_1

PFOS $(100 \mu \mathrm{M})$

U0126 $(10 \mu \mathrm{M})$ -

$\begin{array}{lll}+ & + & - \\ - & + & +\end{array}$

p-ERK1:

p-ERK2 $\stackrel{\overrightarrow{43}}{\longrightarrow}$

$\beta$-actin $\stackrel{43}{\rightarrow} \square$

$1 \mathrm{~h}$

Beclin $\underset{55}{\longrightarrow}$

LC3B-I

$\mathrm{LC} 3 \mathrm{~B}-\mathrm{II} \stackrel{\overline{17}}{\rightarrow}$

p62 
Fig 5A_2 PFOS $(100 \mu \mathrm{M}) \quad-$

$\mathrm{U} 0126(10 \mu \mathrm{M}) \quad$ -

$+\quad+$

$\begin{array}{lll}- & +\end{array}$

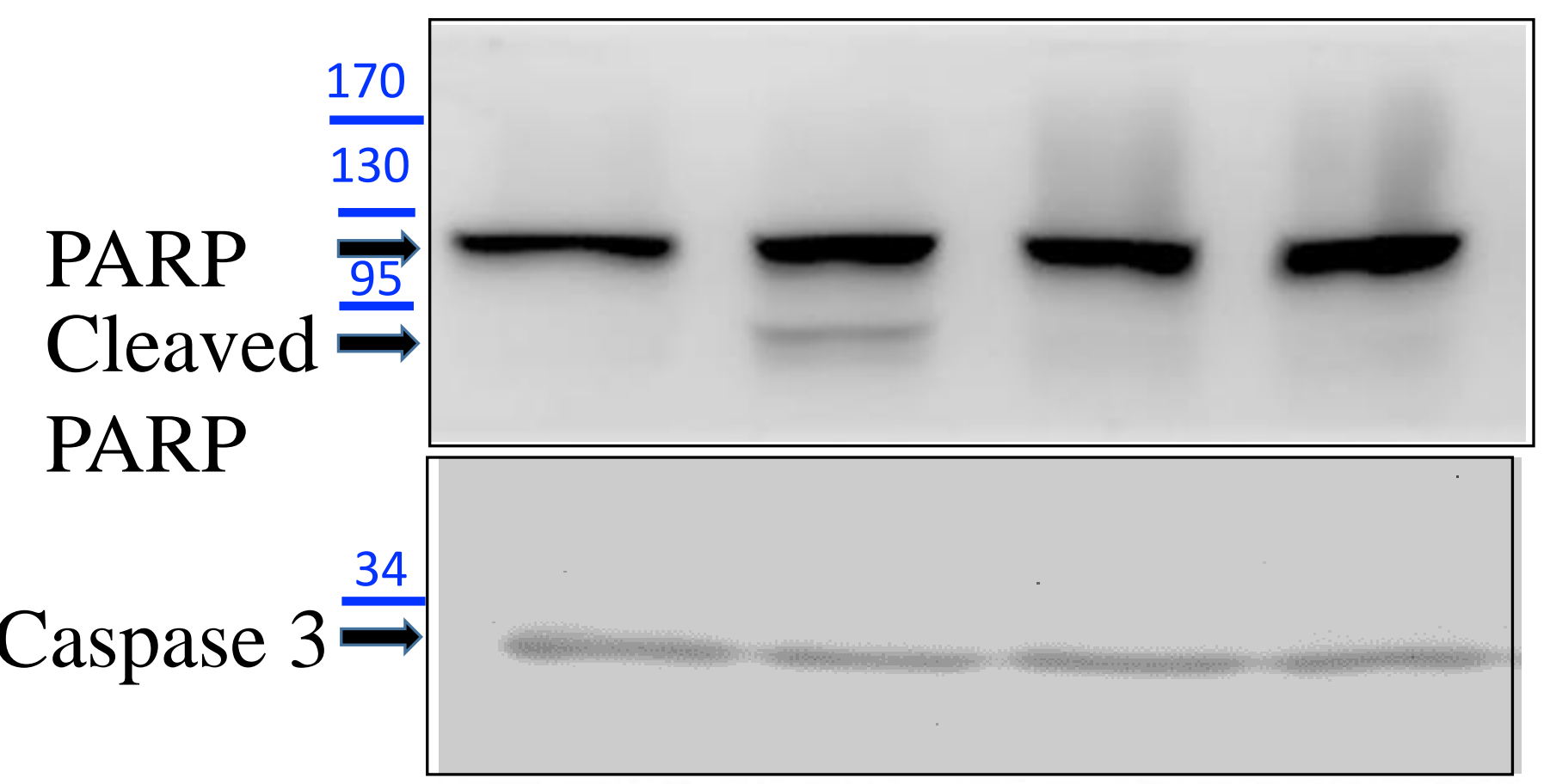

Caspase $3 \stackrel{34}{\Rightarrow}$

Cleaved

26

caspase $3^{\overline{17}}$

$\beta$-actin 
Fig 5B_1

PFOS $(100 \mu \mathrm{M})^{-}$

$+$

NAC (10mM) -

$-\quad+$

$+$

p-ERK1

p-ERK2 $\stackrel{43}{\longrightarrow}$

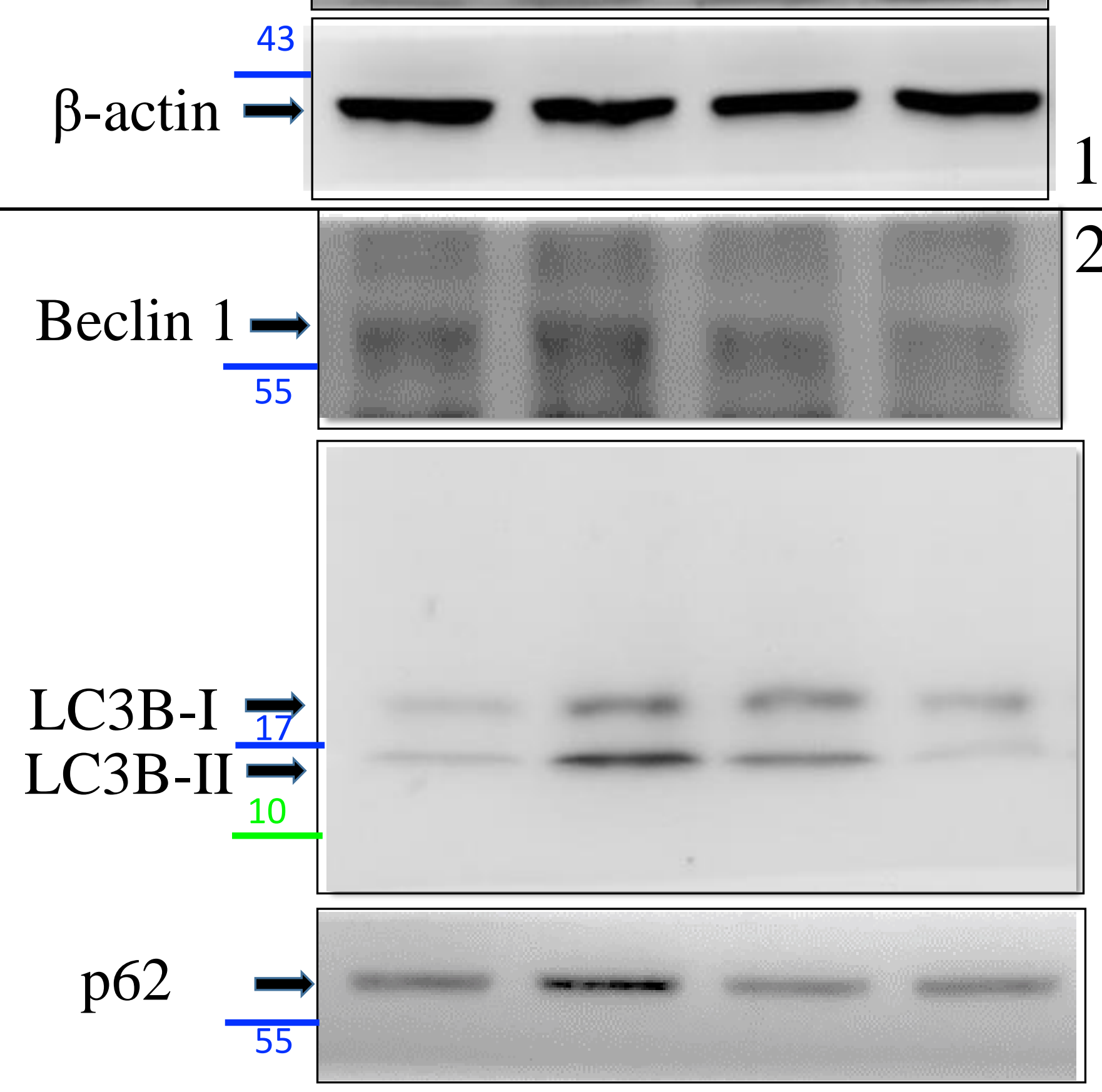

$1 \mathrm{~h}$

$24 \mathrm{~h}$ 
Fig 5B_2

$24 \mathrm{~h}$

$\begin{array}{lllll}\text { PFOS }(100 \mu \mathrm{M}) & - & + & + & - \\ \mathrm{NAC}(10 \mathrm{mM}) & - & - & + & +\end{array}$

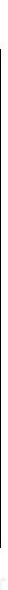

Caspase $3 \underset{34}{\longrightarrow}$

Cleaved

caspase 317

10

$\beta$-actin $\stackrel{43}{=}$ 